Pacific Journal of Mathematics

CONVOLUTION SEMIGROUPS ON HYPERGROUPS 


\title{
CONVOLUTION SEMIGROUPS ON HYPERGROUPS
}

\author{
RUPERT LASSER \\ Dedicated to Professor Elmar Thoma on the occasion of his 60 th birthday
}

\begin{abstract}
The purpose of this paper is to establish a unified treatment of many disparate theorems of Levy-Hincin type. The appropriate framework to do this is the theory of commutative hypergroups. In this way we not only generalize the results mentioned above but also settle some asymmetries indicated above. Roughly speaking a hypergroup $K$ is a space in which the product of two elements is a probability measure on this space satisfying certain conditions. If $K$ is commutative and if the space $\hat{K}$ of characters is a hypergroup under pointwise operations a Levy-Hinčin formula for convolution semigroups is obtained. Before setting up some notation we show how the examples fit in.
\end{abstract}

Introduction. Continuous convolution semigroups $\left(\mu_{t}\right)_{t \geq 0}$ on locally compact abelian groups $G$ are described completely by the so-called Levy-Hinčin formula, see [1] and [6]. This is done by a canonical representation of the negative definite function $\psi$ on $\hat{G}$, which is in one-to-one correspondence to $\left(\mu_{t}\right)_{t \geq 0}$. Beyond that there exist theorems of LevyHinčin type for convolution semigroups built up in various ways. The following subsumes some approaches to this subject.

For certain Gelfand pairs $(G, H)$ convolution semigroups consisting of $H$-biinvariant probability measures $\mu_{t}$ on $G$ allow a Levy-Hinčin representation. In this situation one studies negative definite functions $\psi$ defined on the set $S^{p}(G, H)$ of all positive definite spherical functions. Negative definiteness on $S^{p}(G, H)$ has to be defined in an appropriate way. A nice summary on this topic containing a lot of references is [11]. As a dual concept a Levy-Hinčin formula for $H$-biinvariant negative definite functions on $G$ is established in [5]. Of course here one misses a corresponding convolution semigroup on $S^{P}(G, H)$.

In [4] Bochner characterized homogeneous stochastic processes associated with ultraspherical polynomials via a Levy-Hinčin formula, see also [7]. The part of the convolution semigroup is played by a semigroup of bounded sequences $\left(c_{n}(t)\right)_{n=0}^{\infty}$ bearing a certain positive definite property. In [13] Kennedy studied homogeneous stochastic processes which may be viewed as dual to the class considered by Bochner. Here the part of the convolution semigroup is played by a semigroup of bounded functions $f_{t}(x),-1 \leq x \leq 1$, bearing a certain positive definite property. 
For a Gelfand pair $(G, H)$ the double coset space $K=G / / H$ is a commutative hypergroup, see $[12,8.2 \mathrm{~B}]$. Now in many cases $\hat{K}$ is a hypergroup and each member of $\hat{K}$ may be identified with a function of $S^{p}(G, H)$ as well as each $H$-biinvariant measure on $G$ may be identified with a measure on $K$. Moreover in many cases the dual of $S^{p}(G, H)=\hat{K}$ is $G / / H=K$ again. Then there is also a connection between $H$-biinvariant negative definite functions on $G$ and convolution semigroups defined on $S^{p}(G, H)=\hat{K}$ via a Bochner theorem. In this manner convolution semigroups with respect to $(G, H)$ or with respect to $S^{p}(G, H)$ have a Levy-Hinčin representation in a unified way.

The stochastic processes studied by Bochner are corresponding via Fourier transformation with a convolution semigroup defined on a hypergroup $K=[-1,1]$. The "dual" stochastic process in [13] is the Fourier transform of a convolution semigroup on a hypergroup $K=\mathbf{N}_{0}=\mathbf{N} \cup$ $\{0\}$. Hypergroup structures on $\mathbf{N}_{0}$ connected with orthogonal polynomials are studied in [14]. In the case of ultraspherical polynomials or more general Jacobi polynomials the dual space $\hat{K}$ of $K=\mathbf{N}_{0}$ is equal to $[-1,1]$, and $\hat{K}$ is a hypergroup with Pontrjagin duality $\hat{K}=K$, see [14]. In this manner the Levy-Hinčin formula of the convolution semigroup corresponding to the stochastic process of Bochner resp. Kennedy is established by the Levy-Hinčin representation based on hypergroups.

In our exposition we follow that of the relevant chapters in [1]. We note that some methods of proof used in the group case are not available for hypergroups. Let $K$ be a locally compact Hausdorff space. $M(K)$ denotes the space of all bounded Radon measures, $M^{1}(K)$ the subset of all probability measures and $p_{x}$ the point measure of $x \in K$. The support of a measure $\mu$ is denoted by $\operatorname{supp} \mu . C(K)$ denotes the space of continuous functions on $K$. The space $K$ is called a hypergroup if the following conditions are satisfied:

(H1) There exists a map: $K \times K \rightarrow M^{1}(K),(x, y) \mapsto p_{x} * p_{y}$, called convolution, which is continuous, where $M^{1}(K)$ bears the vague topology. The linear extension to $M(K)$, see [12, Lemma 2.4B], satisfies $p_{x} *\left(p_{y} * p_{z}\right)=\left(p_{x} * p_{y}\right) * p_{z}$.

(H2) $\operatorname{supp} p_{x} * p_{y}$ is compact.

(H3) There exists a homeomorphism $K \rightarrow K, x \mapsto \bar{x}$, called involution, such that $x=\overline{\bar{x}}$ and $\left(p_{x} * p_{y}\right)^{-}=p_{\bar{y}} * p_{\bar{x}}$.

(H4) There exists an element $e \in K$, called unit element, such that $p_{e} * p_{x}=p_{x} * p_{e}=p_{x}$.

(H5) $e \in \operatorname{supp} p_{x} * p_{\bar{y}}$ if and only if $x=y$. 
(H6) The map $(x, y) \mapsto \operatorname{supp} p_{x} * p_{y}$ of $K \times K$ into the space of nonvoid compact subsets of $K$ is continuous, the latter space with the topology as given in $[12,2.5]$.

Many examples are contained in [3], [12], [14], [19].

In this paper we only deal with commutative hypergroups, i.e. $p_{x} * p_{y}$ $=p_{y} * p_{x}$. Then $K$ always has a Haar measure, [22]. Denote

$$
\begin{array}{r}
\hat{K}=\left\{\alpha \in C(K): \alpha \neq 0, \text { bounded, } p_{x} * p_{y}(\alpha)=\alpha(x) \alpha(y),\right. \\
\alpha(\bar{x})=\overline{\alpha(x)}\} .
\end{array}
$$

Equipped with the topology of uniform convergence on compacta $\hat{K}$ is a locally compact Hausdorff space. For basic results of Fourier analysis we refer to [12], where hypergroups are called "convos". We shall say that $\hat{K}$ is a hypergroup with respect to pointwise multiplication, if for $\alpha, \gamma \in \hat{K}$ there exists a measure $p_{\alpha} * p_{\gamma} \in M^{1}(\hat{K})$ such that $\alpha(x) \gamma(x)=$ $\int_{\hat{K}} \tau(x) d p_{\alpha} * p_{\gamma}(\tau)$ for each $x \in K$, and $\hat{K}$ is a hypergroup with this convoltuion and complex conjugation as involution and the constant function 1 as unit. In general the dual $\hat{K}$ is not a hypergroup. If $\hat{K}$ is a hypergroup with respect to pointwise multiplication, then $K \subseteq K^{\wedge}$ in a natural manner, [12, Theorem 12.4B]. If in addition $K=K^{\wedge \wedge}$ holds, we shall call $K$ a strong hypergroup.

1. Negative definite functions. Let $K$ be a commutative hypergroup. We note that for many results in this section commutativity is not really used. A continuous function $\psi: K \rightarrow \mathrm{C}$ is called negative definite if for any $x_{1}, \ldots, x_{n} \in K, c_{1}, \ldots, c_{n} \in \mathbf{C}$

$$
\sum_{i, j=1}^{n}\left(\psi\left(x_{i}\right)+\overline{\psi\left(x_{j}\right)}-p_{x_{i}} * p_{x_{j}}(\psi)\right) c_{i} \bar{c}_{j} \geq 0
$$

For example each constant function $c 1, c \geq 0$, is negative definite. Obviously the following holds for a negative definite function $\psi$ :

$$
\begin{gathered}
\psi(e) \geq 0, \quad p_{x} * p_{\bar{x}}(\psi) \in \mathbf{R}, \quad \overline{\psi(x)}=\psi(\bar{x}), \\
\psi(x)+\psi(\bar{x}) \geq p_{x} * p_{\bar{x}}(\psi) .
\end{gathered}
$$

We note that $\operatorname{Re} \psi$ is in general not a nonnegative function, see the remark below. The following statement and its corollary can be proved exactly as in [1, Proposition 7.5, Corollary 7.6 and 7.7].

Proposition 1.1. A function $\psi: K \rightarrow \mathbf{C}$ is negative definite if and only if the following conditions are satisfied:

(a) $\psi$ is continuous, $\psi(e) \geq 0, \overline{\psi(x)}=\psi(\bar{x})$ for each $x \in K$ and 
(b) if $x_{1}, \ldots, x_{n} \in K, c_{1}, \ldots, c \supsetneqq \in \mathbf{C}$ with $\sum_{i=1}^{n} c_{i}=0$, then

$$
\sum_{i, j=1}^{n} p_{x_{i}} * p_{x_{j}}(\psi) c_{i} \bar{c}_{j} \leq 0
$$

holds.

COROLlary 1.2. Let $\psi$ be a function on $K$. nite.

(a) If $\psi$ is negative definite, then $x \mapsto \psi(x)-\psi(e)$ is negative defi-

(b) If $\psi$ is positive definite, then $x \mapsto \psi(e)-\psi(x)$ is negative definite.

REMARK. Consider $K=\mathbf{N}_{0}$ equipped with a hypergroup structure as defined in [14, §2]. This hypergroup $\mathbf{N}_{0}$ is intimately connected with a certain orthogonal polynomial sequence $\left\{P_{n}(x)\right\}$. In fact given $x \in \mathbf{R}$ every function $\varphi_{x}: \mathbf{N}_{0} \rightarrow \mathbf{R}, \varphi_{x}(n)=P_{n}(x)$ has the property that $p_{n} * p_{m}\left(\varphi_{x}\right)=\varphi_{x}(n) \varphi_{x}(m)$ for each $n \in \mathbf{N}_{0}$. Thus $\varphi_{x}$ is a positive definite function on the hypergroup $\mathbf{N}_{0}$. By Corollary 1.2(b) $\psi_{x}(n)=1-P_{n}(x)$ is negative definite. If $x>1$ we have $P_{1}(x)>1$. Hence $\psi_{x}(1)<0$.

Proposition 1.3. Let $\psi: K \rightarrow \mathbf{C}$ be a negative definite function on $K$.

(a) If $\operatorname{Re} \psi \geq 0$ holds, then $\operatorname{Re} \psi \geq \psi(e) \geq 0$.

(b) If $\psi$ is bounded, then $\operatorname{Re} \psi \geq \psi(e) \geq 0$.

Proof. Given $x \in K$ choose $x_{1}=e, x_{2}=x$ and $c_{1}=1, c_{2}=-1$. By Proposition 1.1 we know that $2 \cdot \operatorname{Re} \psi(x) \geq \psi(e)+p_{x} * p_{\bar{x}}(\psi)$. Now $p_{x} * p_{\bar{x}}(\psi) \in \mathbf{R}$ and $x \mapsto p_{x} * p_{\bar{x}}(\psi)$ is a continuous function on $K$. Thus we obtain

$$
\operatorname{Re} \psi(x) \geq(1 / 2) \psi(e)+\left(1 / 2^{2}\right) \psi(e)+\left(1 / 2^{2}\right) \int_{K} p_{y} * p_{\bar{y}}(\psi) d p_{x} * p_{\bar{x}}(y)
$$

Continuing in this fashion we have

$$
\begin{aligned}
\operatorname{Re} \psi(x) \geq & \left(\sum_{k=1}^{n} 1 / 2^{k}\right) \psi(e) \\
& +\left(1 / 2^{n}\right) \int_{K} \cdots \int_{K} p_{x_{n-1}} * p_{\overline{x_{n-1}}}(\psi) \cdots d p_{x_{1}} \\
& * p_{\bar{x}_{1}}\left(x_{2}\right) d p_{x} * p_{\bar{x}}\left(x_{1}\right) .
\end{aligned}
$$

If $\operatorname{Re} \psi \geq 0$, we know that $p_{x} * p_{\bar{x}}(\psi) \geq 0$ for each $x \in K$. Therefore $\operatorname{Re} \psi \geq\left(\sum_{k=1}^{n} 1 / 2^{k}\right) \psi(e)$ for each $n \in \mathbf{N}$ and then $\operatorname{Re} \psi \geq \psi(e)$. If $\psi$ is bounded, the absolute value of the $(n-1)$-fold integral above is less than $\|\psi\|_{u}$. This implies again that $\operatorname{Re} \psi \geq \psi(e)$. 
Proposition 1.4. Let $\psi: K \rightarrow \mathbf{C}$ be a positive definite function with $\operatorname{Re} \psi \geq 0$. Then $\sqrt{\left|p_{x} * p_{y}(\psi)\right|} \leq \sqrt{|\psi(x)|}+\sqrt{|\psi(y)|}$ for $x, y \in K$.

Proof. A small modification of the arguments in [1, Proposition 7.15] yields that

$$
\begin{aligned}
\mid \psi(x)+\overline{\psi(y)} & -\left.p_{x} * p_{y}(\psi)\right|^{2} \\
& \leq\left(2 \operatorname{Re} \psi(x)-p_{x} * p_{\bar{x}}(\psi)\right)\left(2 \operatorname{Re} \psi(y)-p_{y} * p_{\bar{y}}(\psi)\right) .
\end{aligned}
$$

Since $p_{x} * p_{\bar{x}}(\psi) \in \mathbf{R}$, we know that

$$
p_{x} * p_{\bar{x}}(\psi)=\int_{K} \operatorname{Re} \psi(y) d p_{x} * p_{\bar{x}}(y) \geq 0 .
$$

Therefore $\left|\psi(x)+\overline{\psi(y)}-p_{x} * p_{\bar{y}}(\psi)\right|^{2} \leq 4|\psi(x) \| \psi(y)|$, and the assertion follows.

TheOREM 1.5. Let $\psi: K \rightarrow \mathrm{C}$ be a function on $K$. Assume that

(a) $\psi$ is continuous and $\psi(e) \geq 0$,

(b) $\varphi_{t}: x \mapsto \exp (-t \psi(x))$ are positive definite for each $t>0$. Then $\psi$ is negative definite.

Proof. By (a) the functions $\varphi_{t}$ are continuous and $\varphi_{t}(e) \leq 1$. Therefore Corollary 1.2(b) implies that $x \mapsto(1 / t)\left(1-\varphi_{t}(x)\right)$ is negative definite for any $t>0$. Since

$$
\left|\psi(x)-(1 / t)\left(1-\varphi_{t}(x)\right)\right| \leq t \exp |\psi(x)| \text { for } 0<t<1,
$$

we obtain that $\lim _{t \rightarrow 0}(1 / t)\left(1-\varphi_{t}\right)=\psi$ uniformly on compact subsets of $K$. Now one can easily prove that $\psi$ satisfies $(N)$.

We do not know whether the inverse multiplication of this theorem does hold in general. Now some general examples of negative definite functions are given. A continuous function $h: K \rightarrow \mathbf{R}$ is called a homomorphism, if $h(\bar{x})=-h(x)$ and $p_{x} * p_{y}(h)=h(x)+h(y)$ for $x, y \in K$. The following assertion is immediate.

Proposition 1.6. If $h: K \rightarrow \mathbf{R}$ is a homomorphism, then $\psi=$ ih is negative definite.

A continuous function $q: K \rightarrow \mathbf{R}$ is called a quadratic form, if

$$
p_{x} * p_{y}(q)+p_{x} * p_{\bar{y}}(q)=2(q(x)+q(y)), \quad x, y \in K .
$$


Obviously a quadratic form $q$ satisfies:

(Q)

$$
\begin{aligned}
& q(e)=0, \quad q(\bar{x})=q(x) \text { and } \\
& \mu * \nu(q)+\mu * \bar{\nu}(q)=2(\nu(q) \mu(K)+\mu(q) \nu(K)), \\
& \mu, \nu \in M(K) .
\end{aligned}
$$

LEMMA 1.7. Let $q$ be a quadratic form, and let $\mu \in M(K)$. Then

$$
\mu^{n}(q)=n^{2} \cdot \mu(K)^{n-1} \mu(q)-(n(n-1) / 2) \mu(K)^{n-2} \mu * \bar{\mu}(q)
$$

for each $n \in \mathbf{N}, n \geq 2$, holds, where $\mu^{n}=\mu * \cdots * \mu$, the $n$-fold convolution of $\mu$.

Proof. We prove the statement by induction on $n$. By (Q) we obtain

$$
\begin{aligned}
\mu^{n+1}(q) & =2\left(\mu^{n}(q) \mu(K)+\mu(K)^{n} \mu(q)\right)-\mu^{n} * \bar{\mu}(q) \text { and } \\
\mu^{n} * \bar{\mu}(q) & =(1 / 2)\left[\mu^{n-1} *(\mu * \bar{\mu})(q)+\mu^{n-1} *(\mu * \bar{\mu})(q)\right] \\
& =\mu^{n-1}(q) \mu(K)^{2}+\mu(K)^{n-1} \mu * \bar{\mu}(q) .
\end{aligned}
$$

Thus by the induction assumption

$$
\begin{aligned}
\mu^{n+1}(q)= & 2 \mu(K)^{n} \mu(q) \\
& +2 \mu(K)\left[n^{2} \mu(K)^{n-1} \mu(q)-(n(n-1) / 2) \mu(K)^{n-2} \mu * \bar{\mu}(q)\right] \\
& -\mu(K)^{2}\left[(n-1)^{2} \mu(K)^{n-2} \mu(q)\right. \\
& \left.\quad-((n-1)(n-2) / 2) \mu(K)^{n-3} \mu * \bar{\mu}(q)\right] \\
& -\mu(K)^{n-1} \mu * \bar{\mu}(q) \quad-\mu(K)^{n}(n+1)^{2} \mu(q)-\mu(K)^{n-1}((n+1) n / 2) \mu * \bar{\mu}(q) .
\end{aligned}
$$

COROllary 1.8.. Let $q$ be a quadratic form. Then

$$
\lim _{n \rightarrow \infty} \frac{p_{x}^{n}(q)}{n^{2}}=q(x)-(1 / 2) p_{x} * p_{\bar{x}}(q) .
$$

In particular if $q$ is nonnegative (i.e. $q(x) \geq 0$ ) then

$$
p_{x} * p_{\bar{x}}(q) \leq 2 q(x) \leq p_{x} * p_{x}(q) \text {. }
$$

COROLlaRY 1.9. The only bounded quadratic form $q$ on $K$ is $q=0$. 
Proof. Since $q$ is a quadratic form, $p_{x}^{n} * p_{\bar{x}}^{n}(q)=n \cdot p_{x} * p_{\bar{x}}(q)$ is satisfied for each $n \in \mathbf{N}, x \in K$. In fact $p_{x}^{n+1} * p_{\bar{x}}^{n+1}(q)=p_{x}^{n} * p_{\bar{x}}^{n}(q)+$ $p_{x} * p_{\bar{x}}(q)$ holds by means of $(\mathrm{Q})$. Now a simple induction argument applies. The boundedness of $q$ implies that $p_{x} * p_{\bar{x}}(q)=0$ for each $x \in K$. Therefore $\lim _{n \rightarrow \infty}=\left(1 / n^{2}\right) p_{x}^{n}(q)=q(x)$. Again by the boundedness of $q$ wee see that $q=0$.

Proposition 1.10. Each nonnegative quadratic form on $K$ is a negative definite function.

Proof. By means of Proposition 1.1 we have to show: if $x_{1}, \ldots, x_{m} \in$ $K, c_{1}, \ldots, c_{m} \in \mathbf{R}$ with $\sum_{i=1}^{m} c_{i}=0\left(c_{i} \neq 0\right)$ and $\lambda=\sum_{i=1}^{m} c_{i} p_{x_{i}} \in M(K)$, then $\lambda * \bar{\lambda}(q) \leq 0$. Let $\lambda=\mu-\nu, \mu=\lambda^{+} \in M^{+}(K), \nu=\lambda^{-} \in M^{+}(K)$, the decomposition of $\lambda$ into its positive parts. In particular $\mu(K)=$ $\sum_{c_{i}>0} c_{i}>0, \nu(K)=-\sum_{c_{i}<0} c_{i}>0$, and consequently $\mu(K)=\nu(K)$. By Lemma 1.7 we know

$$
\begin{aligned}
& n^{2} \mu * \nu(K)^{n-1} \mu * \bar{\nu}(q) \\
& \quad=\mu^{n} * \bar{\nu}^{n}(q)+(n(n-1) / 2) \mu * \bar{\nu}(K)^{n-2} \mu * \bar{\mu} * \nu * \bar{\nu}(q)
\end{aligned}
$$

for each $n \in \mathbf{N}$. Further by (Q)

$$
\mu * \bar{\mu} * \nu * \bar{\nu}(q)=\mu * \bar{\mu}(q) \nu * \bar{\nu}(K)+\nu * \bar{\nu}(q) \mu * \bar{\mu}(K) .
$$

Therefore

$$
\begin{aligned}
\mu * \bar{\nu}(q)= & \frac{\mu^{n} * \bar{\nu}^{n}(q)}{n^{2} \mu(K)^{n-1} \nu(K)^{n-1}} \\
& +\frac{\mu * \bar{\mu}(q) \nu(K)^{2}+\nu * \bar{\nu}(q) \mu(K)^{2}}{\mu(K) \nu(K)}(1 / 2-1 /(2 n))
\end{aligned}
$$

for each $n \in \mathbf{N}$. Consequently

$$
\begin{aligned}
\mu * \bar{\nu}(q)-(1 / 2) \mu * \bar{\mu}(q)-(1 / 2) \nu * \bar{\nu}(q) \\
=\lim _{n \rightarrow \infty} \frac{\mu^{n} * \bar{\nu}^{n}(q)}{n^{2} \mu(K)^{n-1} \nu(K)^{n-1}} \geq 0 .
\end{aligned}
$$

Thus we have established that

$$
\lambda * \bar{\lambda}(q)=\mu * \bar{\mu}(q)-2 \mu * \bar{\nu}(q)+\nu * \bar{\nu}(q) \leq 0 .
$$

EXAMPLES. We conclude this section by calculating nonnegative quadratic forms on $K=\mathbf{N}_{0}$ with a hypergroup structure as defined in [14, §] and yet mentioned in the remark above. Since in this situation the 
identity map is the involution on $K=\mathbf{N}_{0}$ the defining property of quadratic forms reduces to $p_{m} * p_{n}(q)=q(m)+q(n)$ for each $m, n \in \mathbf{N}_{0}$. The only homomorphism is the null-function. Let $\left(a_{n}\right),\left(b_{n}\right),\left(c_{n}\right)$ be the sequences, which define the convolution on $\mathbf{N}_{0},[14, \S 2]$. Define recursively the following sequence $\left(s_{n}\right)$ :

$$
s_{0}=0, \quad s_{1}=1, \quad s_{n+1}=\left(1 / a_{n}\right)\left(1+\left(1-b_{n}\right) s_{n}-c_{n} s_{n-1}\right)
$$

for $n=2,3, \ldots$ We note that $\left(s_{n}\right)$ is an increasing sequence. In fact $s_{n+1}-s_{n}=\left(1 / a_{n}\right)\left(1+c_{n}\left(s_{n}-{ }_{n-1}\right)\right)$, since $a_{n}+b_{n}+c_{n}=1$. By induction we see that $s_{n+1} \geq s_{n}$.

Proposition 1.11. Let $K=\mathbf{N}_{0}$ be a hypergroup defined by the sequences $\left(a_{n}\right),\left(b_{n}\right),\left(c_{n}\right)$ as in $[14, \S 2]$. Denote by $\left(s_{n}\right)$ the sequence from (i). Then the nonnegative quadratic forms on $K=\mathbf{N}_{0}$ are exactly the functions $q(n)=s_{n} \cdot a$, where $a \geq 0$.

Proof. Assume that $q: \mathbf{N}_{0} \rightarrow \mathbf{R}$ is a nonnegative quadratic form. Then $q(0)=0$. Let $a=q(1) \geq 0$. Since $p_{1} * p_{n}=a_{n} p_{n+1}+b_{n} p_{n}+c_{n} p_{n-1}$, $n \in \mathbf{N}$ see [14], we have $q(n+1)=\left(1 / a_{n}\right)\left(q(1)+\left(1-b_{n}\right) q(n)\right.$ $-c_{n} q(n-1)$. Now by induction it follows that $q(n)=s_{n} \cdot a$. Conversely we show that for $a \geq 0$ the function $q(n)=s_{n} \cdot a$ is a nonnegative quadratic form. Since $\left(s_{n}\right)$ is increasing $q$ is nonnegative. We use induction on $m$ to prove that $p_{m} * p_{n}(q)=q(m)+q(n)$ for each $m, n \in \mathbf{N}_{0}$, $m \leq n$. For $m=1$ this equation follows by the definition of $s_{n}$. Let $m+1 \leq n$. By the induction assumption we see that

$$
\begin{aligned}
p_{m+1} * p_{n}(q)= & \left(1 / a_{m}\right) p_{1} *\left(p_{m} * p_{n}\right)(q)-\left(b_{m} / a_{m}\right) p_{m} * p_{n}(q) \\
& -\left(c_{m} / a_{m}\right) p_{m-1} * p_{n}(q) \\
= & \left(1 / a_{m}\right)(q(1)+q(m)+q(n)) \\
& -\left(b_{m} / a_{m}\right)(q(m)+q(n))-\left(c_{m} / a_{m}\right)(q(m-1)+q(n)) \\
= & q(n)+q(m+1) .
\end{aligned}
$$

For the prominent case, where $K=\mathbf{N}_{0}$ bears the convolution structure which is "realized" by the ultraspherical polynomials, i.e. $a_{n}=$ $(n+1+2 \alpha) /(2 n+1+2 \alpha), b_{n}=0, c_{n}=n /(2 n+1+2 \alpha), \alpha \geq-1 / 2$, see $[14, \S 3(\mathrm{a})]$, we can calculate $s_{n}$ explicitly:

$$
s_{n}=\frac{n(n+1+2 \alpha)}{2+2 \alpha} \text {. }
$$

This follows by a direct induction argument. 
REMARK. Let $K=G / / H$, a double coset space, where $G$ is a locally compact group, $H$ a compact subgroup of $G$. An $H$-biinvariant function on $G$ may be viewed as a function on $K$. It is immediate that every (continuous) $H$-biinvariant negative definite function on the group $G$ is a negative definite function on the hypergroup $G / / H$. In [10] Harzallah has noted that there exist a double coset space $G / / H$ and a $H$-biinvariant function on $G$, which is a nonnegative quadratic form on the hypergroup $K=G / / H$, but is not a negative definite function on the group $G$. By Proposition 1.10 we see that a $H$-biinvariant function on $G$, which is negative definite on the hypergroup $K=G / / H$, is in general not negative definite on the group $G$.

2. Convolution semigroups. Let $K$ again be a commutative hypergroup. A family $\left(\mu_{t}\right)_{t>0}, \mu_{t} \in M^{+}(K)$ is called a convolution semigroup on $K$, if

(a) $\mu_{t}(K) \leq 1$ for each $t>0$,

(b) $\mu_{t} * \mu_{s}=\mu_{t+s}$ for $t, s>0$,

(c) $\lim _{t \rightarrow 0} \mu_{t}=p_{e}$ with respect to the vague topology on $M(K)$.

LEMMA 2.1. Let $\left(\mu_{t}\right)$ be a convolution semigroup on $K$. Then for $\alpha \in \hat{K}$ the function $t \mapsto \hat{\mu}_{t}(\alpha), \mathbf{R}^{+} \rightarrow \mathbf{C}$ is continuous.

Proof. Obviously it is sufficient to show that $\lim _{t \rightarrow 0} \hat{\mu}_{t}(\alpha)=1$. By [1, Proposition 1.4] we have to show that $\lim _{t \rightarrow 0} \mu_{t}(K)=1$. This follows by the argument of [1, Proposition 8.2].

As one might expect one can study convolution semigroups on $K$ by means of negative definite functions on $\hat{K}$. Of course we have to assume that $\hat{K}$ is a hypergroup with respect to pointwise multiplication.

THEOREM 2.2. Assume that $\hat{K}$ is a hypergroup with respect to pointwise multiplication. If $\left(\mu_{t}\right)_{t>0}$ is a convolution semigroup on $K$, then there exists exactly one negative definite function $\psi: \hat{K} \rightarrow \mathbf{C}$ with $\operatorname{Re} \psi \geq 0$ such that

$$
\hat{\mu}_{t}(\alpha)=\exp (-t \psi(\alpha)) \text { for each } \alpha \in \hat{K}, \quad t>0 .
$$

Proof. One has to give some obvious modifications to the proof of [1, Theorem 8.3].

The negative definite function $\psi: \hat{K} \rightarrow \mathbf{C}$ with $\operatorname{Re} \psi \geq 0$ defined in the above theorem is called associated to $\left(\mu_{t}\right)$. Unfortunately we can only prove a rather weak converse implication of Theorem 2.2. 
THeOREM 2.3. Let $K$ be a strong hypergroup. Let $\psi: \hat{K} \rightarrow \mathbf{C}$ be a negative definite function with $\operatorname{Re} \psi \geq 0$, such that $\psi_{t}(\alpha)=\exp (-t \psi(\alpha))$ is positive definite for $t>0$. There exists a unique convolution semigroup $\left(\mu_{t}\right)$ on $K$ such that $\psi$ is associated to $\left(\mu_{t}\right)$.

Proof. Since $\operatorname{Re} \psi \geq 0$ we know that $\mid \exp (-t \psi(\alpha) \mid \leq 1$. Thus using $K=\hat{\hat{K}}$ by [12, Theorem 12.3B] there are unique determined measures $\mu_{t} \in M^{+}(K), t>0$, such that $\hat{\mu}_{t}(\alpha)=\exp (-t \psi(\alpha))$. Obviously $\left(\mu_{t}\right)$ satisfies the properties (a), (b); use [21, Theorem II.5.5]. Further using the boundedness of $\psi$ on compact subsets of $\hat{K}$, we know

$$
\lim _{t \rightarrow 0} \hat{\mu}_{t}(\alpha)=\lim _{t \rightarrow 0} \exp (-t \psi(\alpha))=1
$$

on compact subsets. Let $f \in C_{00}(K), \varepsilon>0$. By [15, Proposition 1.1], or using $K=\hat{\hat{K}}$ and [12, Theorem 7.3H], there exists a function $g \in C_{00}(\hat{K})$ such that $\|f-\check{g}\|_{u}<\varepsilon$. Now we obtain

$$
\left|\mu_{t}(f)-p_{e}(f)\right| \leq 2 \varepsilon+\int_{\hat{K}}|g(\alpha)|\left|\hat{\mu}_{t}(\bar{\alpha})-1\right| d \pi(\alpha) .
$$

Therefore $\lim _{t \rightarrow 0} \mu_{t}=p_{e}$ in the vague topology on $M(K)$.

EXAMPLE. Fix $\alpha \geq-1 / 2$. We consider the quadratic forms $q(n)=$ $s_{n} \cdot a, a \geq 0$, on the "ultraspherical hypergroup" $\mathbf{N}_{0}$ to $\alpha$, where $s_{n}$ is given by the formula (ii) in $\$ 1$. This hypergroup is a strong one, see [14, \$4]. We give a convolution semigroup $\left(\mu_{t}\right)$ on the dual hypergroup $\widehat{\mathbf{N}_{0}}=[-1,1]$ such that $q$ is associated to $\left(\mu_{t}\right)$. We call this $\left(\mu_{t}\right)$ the Brownian semigroup on $[-1,1]=\widehat{\mathbf{N}}_{0}$. Our arguments are simple modifications of those of [5]. First we note for arbitrary $\mu \in M^{+}(K)$ that $\exp (t \hat{\mu})$, $t>0$, is positive definite on $\hat{K}$. In fact $(\hat{\mu})^{n}=\left(\mu^{n}\right)^{r}$ and $\mu^{n} \in M^{+}(K)$. Using the exponential power series we see that $\exp (t \hat{\mu})$ is positive definite. Therefore $\exp (-t(1-\hat{\mu}))$ is positive definite. Let $\left\{P_{n}^{\alpha}(x)\right\}$ be the ultraspherical polynomial sequence. In particular we know, that

$$
n \mapsto \exp \left(-t\left(1-P_{n}^{\alpha}(x)\right)\right)
$$

is positive definite for each $x \in[-1,1]$. Let $x \in[-1,1[$. Replace $t$ by $t(1-x)^{-1}$ obtaining that $n \mapsto \exp \left(-t\left(1-P_{n}^{\alpha}(x)\right)(1-x)^{-1}\right)$ is positive definite. Now

$$
\lim _{x \rightarrow 1}\left(1-P_{n}^{\alpha}(x)\right)(1-x)^{-1}=n(n+2 \alpha+1) /(2+2 \alpha)
$$

see $[4,2.2 .19]$. It follows that $n \mapsto \exp (-t q(n))$ is positive definite. Now Theorem 2.3 yields the Brownian semigroup on $[-1,1]$.

3. The Lévy-Hinčin representation. We assume throughout this section that $K$ is a commutative hypergroup such that $\hat{K}$ is a hypergroup with respect to pointwise multiplication. Let $S=\left\{\mu \in M^{1}(K): \mu=\right.$ $\bar{\mu}, \operatorname{supp} \mu$ compact $\}$. 
LEMMA 3.1. Let $V$ be a compact neighbourhood of $e \in K$. Then there exists $a \sigma \in S$ such that $-1 / 2 \leq \check{\sigma}(x) \leq 1 / 2$ for each $x \in K \backslash V$.

Proof. There exists a compact neighbourhood $U$ of $e$ such that $\bar{U}=U, U * U \subseteq V$. Let $\varphi=1 / m(U) \chi_{U} * \chi_{U}$, where $\chi_{U}$ is the characteristic function of $U$, and $m$ the Haar measure on $K$. We know that $\varphi$ is a positive definite function on $K$ such that $0 \leq \varphi \leq 1, \varphi(e)=1, \varphi(x)=0$ for $x \in K \backslash V$, see [12, Lemma 6.2E and Lemma 11.3A]. By [12, Theorem $12.3 \mathrm{~B}]$ there exists a $\mu \in M^{+}(\hat{K})$ such that $\check{\mu}=\varphi$. One easily obtains that $\mu \in M^{1}(\hat{K})$ and $\mu=\bar{\mu}$. Choose a compact subset $C \subseteq \hat{K}$ such that $\bar{C}=C$ and $\mu(C) \geq 3 / 4$. Then for $\sigma=(\mu \mid C) / \mu(C) \quad\|\varphi-\check{\sigma}\|\left\|_{u} \leq\right\| \mu-\sigma \| \leq$ $1 / 2$ and thus $-1 / 2 \leq \check{\sigma}(x) \leq 1 / 2$ for $x \in K \backslash V$.

The following statement is contained in the proof of Levy's continuity theorem in [2, Theorem 4.6].

Proposition 3.2. Let $f$ be a bounded continuous function on $\hat{K}$. Let $\left(\mu_{\alpha}\right)$ be a net of positive measures in $M(K)$ such that $\lim \widehat{\mu_{\alpha}} \pi=f \pi$ vaguely ( $\pi$ the Haar measure on $\hat{K}$ ) and $\lim \widehat{\mu_{\alpha}}(1)=f(1)$. Then there exists $a$ $\mu \in M^{+}(K)$ such that $\hat{\mu}=f$ and $\lim \mu_{\alpha}=\mu$ weakly.

Proposition 3.3. Let $\left(\mu_{t}\right)$ be a convolution semigroup on $K$ and $\psi: \hat{K} \rightarrow \mathbf{C}$ the negative definite function associated to $\left(\mu_{t}\right)$. The net $\left((1 / t) \cdot \mu_{t} \mid K \backslash\{e\}\right)_{t>0}$ converges vaguely as $t \rightarrow 0$ to a positive measure $\mu$ on $K \backslash\{e\}$. For each $\sigma \in S$ the function $\psi * \sigma-\psi$ is positive definite and bounded. There exists a measure $\mu_{\sigma} \in M^{+}(K)$ such that $\widehat{\mu_{\sigma}}=$ $\psi * \sigma-\psi$. These measures satisfy

$$
(1-\check{\sigma}) \mu-\mu_{\sigma} \mid K \backslash\{e\} \quad \text { for } \sigma \in S .
$$

The equation ( $\mathrm{L})$ determines $\mu$ uniquely.

Proof. Let $\sigma \in S$. Slightly modifying the proof of [1, Proposition 18.2] we obtain for $\alpha \in \hat{K}$ :

$$
\begin{array}{r}
(1 / t)\left[(1-\check{\sigma}) \mu_{t}\right](\alpha)=(1 / t)\left[\widehat{\mu_{t}}(\alpha)-\int_{\hat{K}} \int_{\hat{K}} \widehat{\mu_{t}}(\gamma) d p_{\alpha} * p_{\bar{\tau}}(\gamma) d \sigma(\tau)\right] \\
=(1 / t)\left[\widehat{\mu_{t}}(\alpha)-\widehat{\mu_{t}} * \sigma(\alpha)\right]=(1 / t)[1-\exp (-t \psi)] *\left(\sigma-p_{1}\right)(\alpha) .
\end{array}
$$

We know that $\lim _{t \rightarrow 0}(1 / t)(1-\exp (-t \psi))=\psi$ uniformly on compact subsets of $\hat{K}$; see the proof of Theorem 1.5. Thus

$$
\lim _{t \rightarrow 0}(1 / t)\left[(1-\check{\sigma}) \mu_{t}\right]^{\dagger}=\psi * \sigma-\psi
$$


uniformly on compact subsets of $\hat{K}$ and $\psi * \sigma-\psi$ is positive definite and bounded. Therefore $\lim _{t \rightarrow 0}(1 / t)\left[(1-\check{\sigma}) \mu_{t}\right]^{\wedge} \pi=(\psi * \sigma-\psi) \pi$ vaguely in the space of all Radon measures on $\hat{K}$. By Proposition 3.2 there exists a measure $\mu_{\sigma} \in M^{+}(K)$ such that $\widehat{\mu_{\sigma}}=\psi * \sigma-\psi$ and

$$
\lim _{t \rightarrow 0}(1 / t)(1-\check{\sigma}) \mu_{t}=\mu_{\sigma}
$$

in the weak topology. The same arguments as in [1, Proposition 18.2] using Lemma 3.1 now show that there exists a positive Radon measure $\mu$ on $K \backslash\{e\}$ such that $\mu=\lim _{t \rightarrow 0}(1 / t) \mu_{t} \mid K \backslash\{e\}$ vaguely and $(1-\check{\sigma}) \mu=\mu_{\sigma} \mid K \backslash\{e\}$ for any $\sigma \in S$. Let $\nu$ be another positive Radon measure on $K \backslash\{e\}$ such that $(1-\check{\sigma}) \mu=(1-\check{\sigma}) \nu$. For $x \in K \backslash\{e\}$ let $\alpha \in \hat{K}$ such that $\operatorname{Re} \alpha(x)<1$. Denote $\sigma=1 / 2\left(p_{\alpha}+p_{\bar{\alpha}}\right) \in S$ and let $V_{x}$ be a compact neighbourhood of $x$ such that $e \notin V_{x}$ and $\check{\sigma} \mid V_{x}<1$. Now it is obvious that $\mu\left|V_{x}=\nu\right| V_{x}$. Thus $\mu=\nu$.

The positive Radon measure $\mu$ on $K \backslash\{e\}$ is called the Levy measure of $\left(\mu_{t}\right)$.

Proposition 3.4. Let $\mu$ denote the Levy measure of a given convolution semigroup $\left(\mu_{t}\right)$. Then

(a) $\int_{K \backslash\{e\}}(1-\operatorname{Re} \gamma(x)) d \mu(x)<\infty$ for each $\gamma \in \hat{K}$,

(b) if $V$ is a compact neighbourhood of $e$ in $K$, then $\mu \mid K \backslash V \in M^{+}(K)$.

Proof. (a) For $\gamma \in \hat{K}$ let $\sigma=1 / 2\left(p_{\gamma}+p_{\bar{\gamma}}\right) \in S$. Then by (L)

$$
\int_{K \backslash\{e\}}(1-\operatorname{Re} \gamma(x)) d \mu(x)=\mu_{\sigma}(K \backslash\{e\})<\infty .
$$

The statement of (b) follows as in [1, Proposition 18.4] using Lemma 3.1.

The following assertions can be proved by an argument as in [1, Lemma 18.13 and Lemma 18.16].

LEMMA 3.5. Let $h: \hat{K} \rightarrow \mathbf{R}$ be continuous and $h(1)=0$. $h$ is a homomorphism if and only if $h * \sigma-h=0$ for each $\sigma \in S$.

LEMMA 3.6. Let $q: \hat{K} \rightarrow \mathbf{R}$ be continuous with $q(\alpha)=q(\bar{\alpha}), q(1)=0$. $q$ is a quadratic form if and only if $q * \sigma-q$ is a constant function for each $\sigma \in S$. Moreover, in the affirmative case $q$ is nonnegative if and only if $q * \sigma-q \geq 0$ for all $\sigma \in S$.

Corollary 3.7. Let $\left(\mu_{t}\right)$ be a convolution semigroup on $K, \psi$ the associated negative definite function. Assume that the Levy measure $\mu$ of $\left(\mu_{t}\right)$ is symmetric. Then $\operatorname{Im} \psi$ is a homomorphism. In particular $i \operatorname{Im} \psi$ is 
negative definite. Further $\mu$ is also the Levy measure of $\left(\nu_{t}\right)$, where $\nu_{t}=\mu_{t / 2} * \overline{\mu_{t / 2}}$.

Proof. $\bar{\mu}=\mu$ is equivalent to $\overline{\mu_{\sigma}}=\mu_{\sigma}$ for each $\sigma \in S$. This is equivalent to $\psi * \sigma-\psi$ being real-valued for each $\sigma \in S$. Thus $(\operatorname{Im} \psi) * \sigma-$ $\operatorname{Im} \psi=0$ for each $\sigma \in S$, and by Lemma 3.5 we know that $\operatorname{Im} \psi$ is a homomorphism. Thus $i \operatorname{Im} \psi$ is negative definite. Further we have seen $(\operatorname{Re} \psi) * \sigma-\operatorname{Re} \psi=\psi * \sigma-\psi$. The proof of Proposition 3.3 yields that $\left(\mu_{t}\right)$ and $\left(\nu_{t}\right)$ define the same class of measures $\mu_{\sigma}, \sigma \in S$. Therefore the uniqueness of the measure satisfying $(\mathrm{L})$ implies the second assertion.

We shall say that $K$ satisfies property (F) if the following holds: If $C \subseteq \hat{K}$ is compact then there exist a constant $M_{C} \geq 0$, a neighbourhood $U_{C}$ of $e$ in $K$ and a finite subset $N_{C}$ of $C$ such that for each $x \in U_{C}$

(F) $\sup \{1-\operatorname{Re} \alpha(x): \alpha \in C\} \leq M_{C} \cdot \sup \left\{1-\operatorname{Re} \alpha(x): \alpha \in N_{C}\right\}$.

Obviously each compact or discrete hypergroup satisfies the property $(F)$. It is known that each locally compact abelian group satisfies property $(F)$, see [18, Lemma 5.1]. At the end of this section we shall give another important class of hypergroups for which property $(\mathrm{F})$ is valid.

Lemma 3.8. Let $K$ satisfy property (F). Assume that $\mu$ is a positive symmetric Radon measure on $K \backslash\{e\}$ such that

$$
\begin{aligned}
& \int_{K \backslash\{e\}}(1-\operatorname{Re} \alpha(x)) d \mu(x)<\infty \text { for each } \alpha \in \hat{K} \text { and } \\
& \mu \mid K \backslash V \in M^{+}(K) \text { for each compact neighbourhood } V \text { of } e .
\end{aligned}
$$

Then the function $\psi_{\mu}: \hat{K} \rightarrow \mathbf{R}, \psi_{\mu}(\alpha)=\int_{K \backslash\{e\}}(1-\operatorname{Re} \alpha(x)) d \mu(x)$ is continuous. Further $\psi_{\mu}$ is negative definite.

Proof. Let $\alpha_{0} \in \hat{K}, \varepsilon>0$ and $C$ a compact neighbourhood of $\alpha_{0}$. Property (F) of $K$ yields $M_{C} \geq 0, N_{C}=\left\{\alpha_{1}, \ldots, \alpha_{n}\right\} \subseteq \hat{K}$ and a neighbourhood $U_{C}$ of $e$ in $K$ such that

$$
\begin{aligned}
\int_{U_{C} \backslash\{e\}} & \sup _{\alpha \in C}(1-\operatorname{Re} \alpha(x)) d \mu(x) \\
\leq & M_{C} \cdot \int_{U_{C} \backslash\{e\}} \sup _{\alpha \in N_{C}}(1-\operatorname{Re} \alpha(x)) d \mu(x) \\
\leq & M_{C} \cdot \sum_{i=1}^{n} \int_{U_{C} \backslash\{e\}}\left(1-\operatorname{Re} \alpha_{i}(x)\right) d \mu(x) \leq M_{C} \cdot \sum_{i=1}^{n} \psi_{\mu}\left(\alpha_{\imath}\right) .
\end{aligned}
$$


Thus there exists a neighbourhood $V$ of $e$ such that

$$
\int_{\bigcap\{e\}}(1-\operatorname{Re} \alpha(x)) d \mu(x)<\varepsilon / 4
$$

for each $\alpha \in C$. Since $\mu \mid K \backslash V$ is bounded there exists a neighbourhood $W_{\alpha_{0}} \subseteq C$ of $\alpha_{0}$ in $\hat{K}$ such that $\left|\int_{K \backslash V}\left(\alpha(x)-\alpha_{0}(x)\right) d \mu(x)\right|<\varepsilon / 2$ for each $\alpha \in W_{\alpha_{0}}$. Thus

$$
\begin{aligned}
\left|\psi_{\mu}(\alpha)-\psi_{\mu}\left(\alpha_{0}\right)\right| \leq & \int_{\bigvee\{e\}}(1-\operatorname{Re} \alpha(x)) d \mu(x) \\
& +\int_{\bigvee\{e\}}\left(1-\operatorname{Re} \alpha_{0}(x)\right) d \mu(x) \\
& +\left|\int_{K \backslash V}\left(\operatorname{Re} \alpha_{0}(x)-\operatorname{Re} \alpha(x)\right) d \mu(x)\right|<\varepsilon
\end{aligned}
$$

for each $\alpha \in W_{\alpha_{0}}$. In order to show that $\psi_{\mu}$ is negative definite we note that for $x \in K$ the function $\tau \mapsto \tau(x)$ is positive definite. Thus $\tau \mapsto 1-$ $\operatorname{Re} \tau(x)$ is negative definite. Given $\alpha, \beta \in \hat{K}$ apply again property (F) to $C=\operatorname{supp} p_{\alpha} * p_{\beta}$ to show that

$$
\int_{K \backslash\{e\}} \sup _{\alpha \in C}(1-\operatorname{Re} \alpha(x)) d \mu(x)<\infty .
$$

Thus we may apply Fubini's theorem leading to the following equation:

$$
\begin{aligned}
\psi_{\mu}(\alpha)+\psi_{\mu}(\beta)-p_{\alpha} * p_{\bar{\beta}}\left(\psi_{\mu}\right) & \\
=\int_{K \backslash\{e\}} & {[(1-\operatorname{Re} \alpha(x))+(1-\operatorname{Re} \beta(x))} \\
& \left.\quad-\int_{\hat{K}}(1-\operatorname{Re} \tau(x)) d p_{\alpha} * p_{\bar{\beta}}(\tau)\right] d \mu(x) .
\end{aligned}
$$

Therefore $\psi_{\mu}$ is negative definite.

Now we can prove a Levy-Hinčin formula.

THEOREM 3.9. Suppose that $K$ satisfies property $(\mathrm{F})$. Let $\left(\mu_{t}\right)$ be a convolution semigroup on $K$ with associated negative definite function $\psi$ : $\hat{K} \rightarrow \mathbf{C}$, and Levy measure $\mu$. Assume that $\mu$ is symmetric.

(a) Then $\psi$ can be written

$$
\psi(\alpha)=c+i h(\alpha)+q(\alpha)+\int_{K \backslash\{e\}}(1-\operatorname{Re} \alpha(x)) d \mu(x),
$$


for $\alpha \in \hat{K}$, where $c$ is a nonnegative constant, $h: \hat{K} \rightarrow \mathbf{R}$ a homomorphism and $q: \hat{K} \rightarrow \mathbf{R}$ a nonnegative quadratic form.

(b) Moreover c, $h, q$ in (*) are determined uniquely by $\left(\mu_{t}\right): c=\psi(1)$, $h=\operatorname{Im} \psi$ and

$$
q(\alpha)=\lim _{n \rightarrow \infty}\left[\frac{p_{\alpha}^{n}(\psi)}{n^{2}}+\frac{p_{\alpha}^{n} * p_{\alpha}^{n}(\psi)}{2 n}\right]
$$

Proof. (a) Let $c=\psi(1)$ and $\psi^{\prime}=\psi-c 1$. Then $\psi^{\prime}$ is a negative definite function associated to a convolution semigroup having the same Levy measure $\mu$ as $\left(\mu_{t}\right)$. Since $\mu$ is symmetric, $h=\operatorname{Im} \psi$ is a homomorphism by Corollary 3.7. Further the function $\psi^{\prime \prime}=\psi-c 1-i$ is negative definite and associated to a convolution semigroup with Levy measure $\mu$. Define $\psi_{\mu}$ as in Lemma 3.8 using Proposition 3.4. Consider $q=\psi^{\prime \prime}-$ $\psi_{\mu}$. The function $q$ is continuous, real-valued, symmetric and $q(1)=0$. Given $\sigma \in S$ we know that $h * \sigma-h=0$ by Lemma 3.5. Thus $\psi^{\prime \prime} * \sigma-$ $\psi^{\prime \prime}=\psi * \sigma-\psi=\widehat{\mu_{\sigma}}$, where the measure $\mu_{\sigma}$ is defined as in Proposition 3.3. Consequently $q * \sigma-q=\widehat{\mu_{\sigma}}-\left(\psi_{\mu} * \sigma-\psi_{\mu}\right)$. One easily obtains that

$$
\begin{aligned}
\psi_{\mu} * \sigma-\psi_{\mu} & =\int_{K \backslash\{e\}} \operatorname{Re} \alpha(x)(1-\check{\sigma}(x)) d \mu(x) \\
& =\int_{K \backslash\{e\}} \overline{\alpha(x)}(1-\check{\sigma}(x)) d \mu(x) .
\end{aligned}
$$

Since $\mu_{\sigma} \mid K \backslash\{e\}=(1-\check{\sigma}) \mu$, we see that $q * \sigma-q=\mu_{\sigma}(\{e\}) \geq 0$. By Lemma 3.6 the function $q$ is a nonnegative quadratic form. Now the statement of (a) is proved.

(b) Of course $c=\psi(1)$ and $h=\operatorname{Im} \psi$. Denote again $\psi_{\mu}(\alpha)=$ $\int_{K \backslash\{e\}}(1-\operatorname{Re} \alpha(x)) d \mu(x)$. We know that $\psi_{\mu}$ is negative definite. By Corollary 1.8 we obtain

$$
\begin{aligned}
q(\alpha) & -(1 / 2) p_{\alpha} * p_{\bar{\alpha}}(q)=\lim _{n \rightarrow \infty} p_{\alpha}^{n}(q) / n^{2} \\
= & \lim _{n \rightarrow \infty} p_{\alpha}^{n}(\psi) / n^{2}-\lim _{n \rightarrow \infty}\left(1 / n^{2}\right) \int_{K \backslash\{e\}}\left(1-\operatorname{Re}\left(\alpha(x)^{n}\right)\right) d \mu(x) .
\end{aligned}
$$

Note that Fubini's theorem is available by property $(F)$. Obviously $\lim _{n \rightarrow \infty}\left(1 / n^{2}\right)\left(1-\operatorname{Re}\left(\alpha(x)^{n}\right)\right)=0$ for each $x \in K$. If $\alpha(x) \neq 0$, let $0<$ $r \leq 1, \theta \in[-\pi, \pi]$ such that $\alpha(x)=r \exp (i \theta)$. Then for $n \in \mathbf{N}$

$$
\begin{aligned}
\left(1 / n^{2}\right)\left(1-\operatorname{Re}\left(\alpha(x)^{n}\right)\right) & =\left(1 / n^{2}\right)\left(1-r^{n}\right)+\left(r^{n} / n^{2}\right)(1-\cos n \theta) \\
& \leq\left(1 / n^{2}\right)\left(1-r^{n}\right)+r^{n} C(1-\cos \theta),
\end{aligned}
$$


where $C>0$ can be established as in [1, p. 183]. Thus

$$
\begin{aligned}
& \left(1 / n^{2}\right)\left(1-\operatorname{Re}\left(\alpha(x)^{n}\right)\right) \leq(1-r)+r^{n-1} C(r-r \cos \theta) \\
& \quad \leq\left(1-r^{2}\right)+C(1-r \cos \theta)=\left(1-|\alpha(x)|^{2}\right)+C(1-\operatorname{Re} \alpha(x)) .
\end{aligned}
$$

For $\alpha(x)=0$ this inequality holds obviously. Since

$$
\begin{gathered}
\int_{K \backslash\{e\}}\left(1-|\alpha(x)|^{2}\right) d \mu(x)+C \cdot \int_{K \backslash\{e\}}(1-\operatorname{Re} \alpha(x)) d \mu(x) \\
=p_{\alpha} * p_{\bar{\alpha}}\left(\psi_{\mu}\right)+C \psi_{\mu}(\alpha)<\infty,
\end{gathered}
$$

the dominated convergence theorem yields that $q(\alpha)=\lim _{n \rightarrow \infty} p_{\alpha}^{n}(\psi) / n^{2}$ $+p_{\alpha} * p_{\bar{\alpha}}(q) / 2$. In the proof of Corollary 1.9 we have noted that $p_{\alpha}^{n} * p_{\bar{\alpha}}^{n}(q)=n \cdot p_{\alpha} * p_{\bar{\alpha}}(q)$ for each $n \in \mathbf{N}$. Thus

$$
\begin{aligned}
p_{\alpha} * & p_{\bar{\alpha}}(q) / 2=\lim _{n \rightarrow \infty} p_{\alpha}^{n} * p_{\bar{\alpha}}^{n}(q) /(2 n) \\
& =\lim _{n \rightarrow \infty} p_{\alpha}^{n} * p_{\bar{\alpha}}^{n}(\psi) /(2 n)-\lim _{n \rightarrow \infty}(1 / 2 n) \int_{K \backslash\{e\}}\left(1-|\alpha(x)|^{2 n}\right) d \mu(x) .
\end{aligned}
$$

Since $(1 / 2 n)\left(1-|\alpha(x)|^{2 n}\right) \leq(1-|\alpha(x)|) \leq\left(1-|\alpha(x)|^{2}\right)$, the dominated convergence theorem applies again. Consequently $p_{\alpha} * p_{\bar{\alpha}}(q) / 2$ $=\lim _{n \rightarrow \infty}(1 / 2 n) p_{\alpha}^{n} * p_{\bar{\alpha}}^{n}(\psi)$.

ExAmples. (a) Consider the hypergroup $K=\mathbf{N}_{0}$ with the structure which corresponds to the Jacobi polynomials $P_{n}^{(\alpha, \beta)}(x), \alpha \geq \beta>-1$, $\alpha+\beta+1 \geq 0$. If we assume that in addition that $\beta \geq-1 / 2$ or $\alpha+\beta \geq 0$ then the dual $\hat{K}$ is a hypergroup and may be identified with $[-1,1]$, see [14, $\S \S 3$ and 4]. Since $\hat{K}$ is compact the homomorphism and the quadratic form in the Levy-Hinčin formula of Theorem 3.9 are zero. Further given a convolution semigroup $\left(\mu_{t}\right)$ on $\mathbf{N}_{0}$ the Levy measure $\mu$ of $\left(\mu_{t}\right)$ is symmetric and bounded. Hence

$$
\begin{aligned}
& \widehat{\mu}_{t}(x)=\exp (-t \psi(x)) \text { for } x \in[-1,1], \text { where } \\
& \psi(x)=c+\sum_{n=1}^{\infty}\left(1-P_{n}^{(\alpha, \beta)}(x)\right) \mu(n) .
\end{aligned}
$$

Compare [13] for the ultraspherical case $\alpha=\beta$.

(b) Consider the dual hypergroup $K=[-1,1]$ corresponding to the Jacobi polynomials $P_{n}^{(\alpha, \beta)}(x)$, where $(\alpha, \beta)$ belongs to the same region as in (a). The dual of $K$ is the hypergroup $\mathbf{N}_{0}$ of (a), see [14, §4]. Given a convolution semigroup $\left(\mu_{t}\right)$ on $[-1,1]$ the Levy measure $\mu$ is symmetric. 
Further the homomorphism is zero. Thus

$$
\begin{aligned}
& \widehat{\mu}_{t}(n)=\exp (-t \psi(n)) \text { for } n \in \mathbf{N}_{0}, \text { where } \\
& \psi(n)=c+q(n)+\int_{-1}^{1-0}\left(1-P_{n}^{(\alpha, \beta)}(x)\right) d \mu(x) \text { and } \\
& q(n)=a s_{n}(a \geq 0), \text { where } s_{n}=\frac{n(n+\alpha+\beta+1)}{\alpha+\beta+2} .
\end{aligned}
$$

The quadratic form $s_{n}$ can be computed by means of formula (i) in Chapter 1. Compare [4] and [7].

(c) We consider now commutative hypergroups which are obtained from certain groups. Let $G$ denote a locally compact group and let $B$ denote a subgroup of the automorphism group $\operatorname{Aut}(G)$ that contains the group $I(G)$ of inner automorphisms. One calls $G$ an $[F I A]_{B}^{-}$group provided the closure $\bar{B}$ of $B$ in $\operatorname{Aut}(G)$ is compact, where $\operatorname{Aut}(G)$ bears the Birkhoff topology. It is easily established that the $\bar{B}$-orbit space $G_{B}$ of $G$ is commutative hypergroup with natural operations, see $[19, \S 1]$. There is an obvious relation between $\bar{B}$-invariant measures or functions on $G$ and measures or functions on $G_{B}$. We note that $G_{B}$ may be identified with the set of all continuous nonzero positive definite $B$-invariant functions $\varphi$ on $G$ which satisfy $\varphi(x) \varphi(y)=\int_{\bar{B}} \varphi(x \beta(y)) d \beta$, where $d \beta$ is the normalized Haar measure on $\bar{B}$, see [16] and [17]. In [9] it is shown that $\widehat{G_{B}}$ is a hypergroup with respect to pointwise multiplication. We prove that $G_{B}$ satisfies property $(\mathrm{F})$.

LEMMA 3.10. Let $K_{1}$ and $K_{2}$ be two hypergroups satisfying property (F). Then $K_{1} \oplus K_{2}$ satisfies property $(\mathrm{F})$.

Proof. Let $0 \leq r_{1}, r_{2} \leq 1$ and $0 \leq x, y \leq 2 \pi$. Then

$$
1-r_{1} r_{2} \cos (x+y) \leq 2\left[\left(1-r_{1} \cos x\right)+\left(1-r_{2} \cos y\right)\right]
$$

is valid. In fact the inequalities $1-r_{1} r_{2} \leq\left(1-r_{1}\right)+\left(1-r_{2}\right)$ and $1-\cos (x+y) \leq 2[(1-\cos x)+(1-\cos y)]$, compare [18, p. 82], imply that

$$
\begin{aligned}
1-r_{1} r_{2} \cos (x & +y)=\left(1-r_{1} r_{2}\right)+r_{1} r_{2}(1-\cos (x+y)) \\
& \leq\left(1-r_{1}\right)+\left(1-r_{2}\right)+2 r_{1}(1-\cos x)+2 r_{2}(1-\cos y) \\
& \leq 2\left[\left(1-r_{1} \cos x\right)+\left(1-r_{2} \cos y\right)\right] .
\end{aligned}
$$

Since each $\alpha \in\left(K_{1} \oplus K_{2}\right)$ is written $\alpha\left(\left(z_{1}, z_{2}\right)\right)=\beta\left(z_{1}\right) \gamma\left(z_{2}\right)$, where $z_{1} \in$ $K_{1}, z_{2} \in K_{2}, \alpha \in \widehat{K_{1}}, \gamma \in \widehat{K_{2}}$, the assertion can be easily proved. 
The structure theorem for $[F I A]_{B}^{-}$groups $G$, see [23], says that $G=V \oplus L, L$ containing an open, compact subgroup $H$, and $V$ being a vector group. By Lemma 3.10 we have to show that $V_{B}$ and $L_{B}$ satisfy property $(\mathrm{F})$. Using [17, Theorem 5.8] we obtain that $V_{B}$ satisfies $(\mathrm{F})$, since $V$ satisfies (F) as an abelian group. The methods of [17, Proposition 2.9] show that the map $r_{H}: \widehat{L_{B}} \rightarrow \widehat{H_{B}}, r_{H}(\alpha)=\alpha \mid H$ is continuous. $\widehat{H_{B}}$ is discrete. Thus for $\alpha \in \widehat{L_{B}}$ the set $C_{\alpha}=\left\{\beta \in \widehat{L_{B}}: \beta|H=\alpha| H\right\}$ is open. This fact implies property $(\mathrm{F})$ for $L_{B}$. Hence $(\mathrm{F})$ holds for $G_{B}$.

Thus Theorem 3.9 characterizes convolution semigroups $\left(\mu_{t}\right)$ on the $[F I A]_{B}^{-}$group $G$ consisting of $B$-invariant measures $\mu_{t}$. The class of $[F I A]_{B}^{-}$groups covers for instance orbit spaces of compact groups or, for $B=I(G)$ of locally compact groups having relatively compact conjugacy classes and having small invariant neighbourhoods of the identity. In particular if $G=\mathbf{R}^{n}, B=\operatorname{SO}(n)$, the special orthogonal group, we obtain the Bessel functions. In fact $G_{B}$ and $\widehat{G_{B}}$ may be identified with $[0, \infty[$. Each non-constant character $\alpha \in \widehat{G_{B}}$ is given by $\left.\alpha=\alpha_{y}, y \in\right] 0, \infty[$, where

$$
\left.\alpha_{y}(x)=\Gamma(\nu+1) 2^{\nu} \frac{J_{\nu}(y x)}{(y x)^{\nu}} \quad \text { for } x \in\right] 0, \infty\left[\text { and } \alpha_{y}(0)=1,\right.
$$

and $J_{\nu}$ is the Bessel function of the first kind of order $\nu$ and $\nu=n / 2-1$. We refer to [20], [5, p. 201] where a Levy-Hinčin formula is established, and [17, Theorem 5.8].

Finally we note that similar arguments yield that $\widehat{G_{B}}$ satisfies property (F), too. The dual of $\widehat{G_{B}}$ is $G_{B}$, see [8], and Theorem 3.9 applies for $K=\widehat{G_{B}}$, $G$ an arbitrary $[F I A]_{B}^{-}$group.

\section{REFERENCES}

[1] C. Berg and G. Forst, Potential Theory on Locally Compact Abelian Groups, Berlin-Heidelberg-New York: Springer 1975.

[2] W. R. Bloom and H. Heyer, The Fourier transform for probability measures on hypergroups, Rend. Mat., 2 (1982), 315-334.

[3] Convergence of convolution products of probability measures on hypergroups, Rend. Mat., 2 (1982), 547-563.

[4] S. Bochner, Sturm-Liouville and heat equations whose eigenfunctions are ultraspherical polynomials or associated Bessel functions, In: Proceedings of the Conference on Differential Equations, pp. 23-48. University of Maryland 1955.

[5] J. Faraut and K. Harzallah, Distances hilbertiennes invariantes sur un espaces homogène. Ann. Inst. Fourier, 24 (1974), 171-217.

[6] G. Forst, The Levy-Hinčin representation of negative definite functions, $\mathrm{Z}$. Wahrscheinlichkeitstheorie verw. Gebiete, 34 (1976), 313-318. 
[7] G. Gasper, Banach algebras for Jacobi series and positivity of a kernel, Ann. of Math., 95 (1972), 261-280.

[8] K. Hartmann, [ FIA] $]_{B}^{-}$Gruppen und Hypergruppen, Mh. Math., 89 (1980), 9-17.

[9] K. Hartmann, R. W. Henrichs and R. Lasser, Duals of orbit spaces in groups with relatively compact inner automorphism groups are hypergroups, Mh. Math., 88 (1979), 229-238.

[10] K. Harzallah, Distances hilbertiennes invariantes sur un espace homogène, In: Théorie du Potentiel et Analyse Harmonique, Lecture Notes in Math. Vol. 404, Springer (1974), 133-137.

[11] H. Heyer, Convolution semigroups of probability measures on Gelfand pairs, Expo. Math., 1 (1983), 3-45.

[12] R. I. Jewett, Spaces with an abstract convolution of measures, Adv. in Math., 18 (1975), 1-101.

[13] M. Kennedy, A stochastic process associated with ultraspherical polynomials, Proc. Royal Irish Acad., 61 Sec. A, (1961), 89-100.

[14] R. Lasser, Orthogonal polynomials and hypergroups, Rend. Math., 3 (1983), 185-209.

[15] _ Fourier-Stieltjes transforms on hypergroups, Analysis, 2 (1982), 281-303.

[16] J. Liukkonen and R. Mosak, Harmonic analysis and centers of group algebras, Trans. Amer. Math. Soc., 195 (1974), 147-163.

[17] R. Mosak, The $L^{1}$-and $C^{*}$-algebras of $[F I A]_{B}^{-}$groups and their representations, Trans. Amer. Math. Soc., 163 (1972), 277-310.

[18] K. R. Parthasarathy, Probability Measures on Metric Spaces, New York-London: Academic Press 1967.

[19] K. A. Ross, Centers of hypergroups, Trans. Amer. Math. Soc., 243 (1978), 251-269.

[20] A. Schwartz, The structure of the algebra of Hankel transforms and the algebra of Hankel-Stieltjes transforms, Canad. J. Math., 23 (1971), 236-246.

[21] R. Spector, Aperçu de la théorie des hypergroupes, In: Analyse Harmonique sur les Groupes de Lie, Lecture Notes in Math. Vol. 497, Springer (1975), 643-673.

[22] _ Mesures invariantes dur les hypergroupes, Trans. Amer. Math. Soc., 239 (1978), 147-165.

[23] T. W. Wilcox, A note on groups with relatively compact conjugacy classes, Proc. Amer. Math. Soc., 42 (1974), 326-329.

Received July 3, 1984.

TECHNISCHE UNIVERSITÄT MÜNCHEN

ARCISSTR. 21

D-8000 MÚNCHEN 2

WEST GERMANY 



\section{PACIFIC JOURNAL OF MATHEMATICS EDITORS}

\author{
V. S. VARADARAJAN \\ (Managing Editor) \\ University of California \\ Los Angeles, CA 90024 \\ HeRbert Clemens \\ University of Utah \\ Salt Lake City, UT 84112 \\ R. FINN \\ Stanford University \\ Stanford, CA 94305
}

\author{
HERMANN FLASCHKA \\ University of Arizona \\ Tucson, AZ 85721 \\ RAMESH A. GANGOLLI \\ University of Washington \\ Seattle, WA 98195 \\ VAUGHAN F. R. JONES \\ University of California \\ Berkeley, CA 94720 \\ ROBION KIRBY \\ University of California \\ Berkeley, CA 94720
}

\author{
C. C. MOORE \\ University of California \\ Berkeley, CA 94720 \\ H. SAMELSON \\ Stanford University \\ Stanford, CA 94305 \\ HAROLD STARK \\ University of California, San Diego \\ La Jolla, CA 92093
}

\section{ASSOCIATE EDITORS}
R. Arens
E. F. BECKENBACH
B. H. NEUMANN
F. WOLF
K. YosHIDA (1906-1982)

\section{SUPPORTING INSTITUTIONS}

UNIVERSITY OF ARIZONA
UNIVERSITY OF BRITISH COLUMBIA
CALIFORNIA INSTITUTE OF TECHN
UNIVERSITY OF CALIFORNIA
MONTANA STATE UNIVERSITY
UNIVERSITY OF NEVADA, RENO
NEW MEXICO STATE UNIVERSITY
OREGON STATE UNIVERSITY

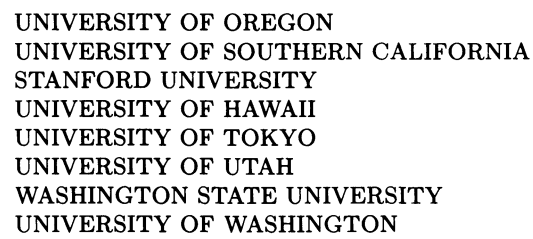

The Supporting Institutions listed above contribute to the cost of publication of this Journal, but they are not owners or publishers and have no responsibility for its content or policies.

Mathematical papers intended for publication in the Pacific Journal of Mathematics should be in typed form or offset-reproduced (not dittoed), double spaced with large margins. Please do not use built up fractions in the text of the manuscript. However, you may use them in the displayed equations. Underline Greek letters in red, German in green, and script in blue. The first paragraph must be capable of being used separately as a synopsis of the entire paper. In particular it should contain no bibliographic references. Please propose a heading for the odd numbered pages of less than 35 characters. Manuscripts, in triplicate, may be sent to any one of the editors. Please classify according to the scheme of Math. Reviews, Index to Vol. 39. Supply name and address of author to whom proofs should be sent. All other communications should be addressed to the managing editor, or Elaine Barth, University of California, Los Angeles, California 90024.

There are page-charges associated with articles appearing in the Pacific Journal of Mathematics. These charges are expected to be paid by the author's University, Government Agency or Company. If the author or authors do not have access to such Institutional support these charges are waived. Single authors will receive 50 free reprints; joint authors will receive a total of 100 free reprints. Additional copies may be obtained at cost in multiples of 50 .

The Pacific Journal of Mathematics is issued monthly as of January 1966. Regular subscription rate: $\$ 190.00$ a year (5 Vols., 10 issues). Special rate: $\$ 95.00$ a year to individual members of supporting institutions.

Subscriptions, orders for numbers issued in the last three calendar years, and changes of address should be sent to Pacific Journal of Mathematics, P.O. Box 969, Carmel Valley, CA 93924, U.S.A. Old back numbers obtainable from Kraus Periodicals Co., Route 100, Millwood, NY 10546.

The Pacific Journal of Mathematics at P.O. Box 969, Carmel Valley, CA 93924 (ISSN 0030-8730) publishes 5 volumes per year. Application to mail at Second-class postage rates is pending at Carmel Valley, California, and additional mailing offices. Postmaster: send address changes to Pacific Journal of Mathematics, P.O. Box 969, Carmel Valley, CA 93924.

PUBLISHED BY PACIFIC JOURNAL OF MATHEMATICS, A NON-PROFIT CORPORATION Copyright (C) 1987 by Pacific Journal of Mathematics 


\section{Pacific Journal of Mathematics \\ Vol. 127, No. $2 \quad$ February, 1987}

Richard Martin Aron and Robert Henry Lohman, A geometric function determined by extreme points of the unit ball of a normed space .... . . 209

Kari Astala and M. S. Ramanujan, $(s)$-nuclear sets and operators $\ldots \ldots \ldots 233$ Earl Robert Berkson, Thomas Alastair Gillespie and Paul Scott Muhly, Analyticity and spectral decompositions of $L^{p}$ for compact abelian

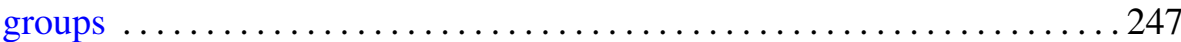

Ronald P. Brown, Real closures of fields at orderings of higher level . . . . . 261

Ronald P. Brown, The behavior of chains of orderings under field

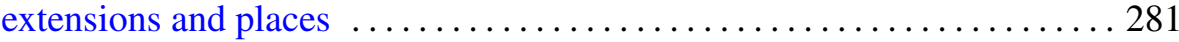

Rodney Graham Downey, Subsets of hypersimple sets .............. 299

Manfred Droste and Saharon Shelah, On the universality of systems of

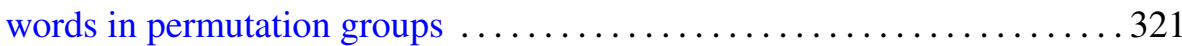

Hidenori Fujiwara, Représentations monomiales des groupes de Lie

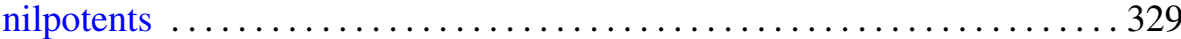

Rupert Lasser, Convolution semigroups on hypergroups $\ldots \ldots \ldots \ldots \ldots 35$

Marcus Marlene Marsh, $u$-mappings on trees $\ldots \ldots \ldots \ldots \ldots \ldots \ldots \ldots \ldots \ldots$

Peter Andrew Symonds, Localization in the classification of flat

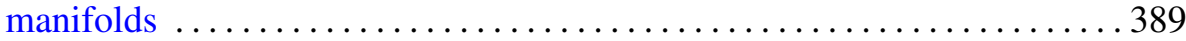

